\title{
Blog institucional del servicio de nefrología "contigo en la enfermedad renal"
}

\author{
María del Mar Salvadores Cabezas, Rosario Aguirremota Corbera, Milagros Menchaca Casin, María Teresa \\ Rosales Monje, María Juana Esparza Pujana, Begoña Parra Barajas
}

Hospital Universitario de Cruces. Bizkaia

\section{Introducción:}

La accesibilidad de la información para pacientes y sus grupos de soporte social, ha aumentado exponencialmente en los últimos años gracias a las TICs (Tecnologías de la Información y Comunicación). Las enfermedades crónicas constituyen hoy una prioridad para las que hay que diseñar estrategias dirigidas al autocuidado. Esta nueva reelaboración de las relaciones clínicas, y el incremento de enfermos crónicos con múltiples patologías asociadas, justifica la creación de un espacio web, "un blog", dirigido a enfermos renales. Movidos por esta realidad socio-sanitaria, profesionales del servicio de Nefrología, iniciamos el proyecto de elaboración de un blog. Tras la revisión bibliográfica (términos de "Social Media", "Social Networks", "Health Care", "Blog", "Web 2.0") y contando con asesores expertos en la gestión de blogs de salud, se ha creado un blog dirigido a enfermos renales. Está desarrollado bajo licencia Creative Commons, con reconocimiento no comercial y en entorno Wordpress. El objetivo es facilitar el acceso a la información de forma ágil, útil, basada en la evidencia científica, disponible cuando y donde se precise. Mediante comentarios y testimonios de los usuarios, queremos abrir nuevos canales de comunicación entre paciente y personal sanitario. Siguiendo las recomendaciones para escribir en la Web, (Centro para el Control y Prevención de Enfermedades), se diseñó el blog que consta de 9 categorías fijas, con 125 páginas que incluyen información sobre fisiopatología renal, tratamientos y hábitos de vida saludables. Hay un espacio para preguntas frecuentes, diccionario, recursos, presentación del equipo de trabajo y enlaces de interés sanitario a otras Webs. Una vez elaborado, se procedió a su divulgación y posterior análisis de las estadísticas de acceso y comentarios recibidos. Periódicamente se generan nuevas entradas con contenidos de interés y actualidad. Desde el 03/12/2012 hasta el 31/03/2013 se recibieron 8.376 visitas. Las páginas más visitadas han sido: ¿quiénes somos?, enfermedad renal y los riñones, preguntas frecuentes, nutrición, hemodiálisis y medicación. Los comentarios son mayoritariamente de pacientes mostrando su agradecimiento y apoyo por esta nueva iniciativa. La realidad de internet como ámbito de interrelación social, está produciendo cambios sustanciales en las relaciones entre pacientes, cuidadores y profesionales sanitarios. Su uso como fuente de información sanitaria se produce cada vez con más frecuencia, y las Webs institucionales son las que merecen mayor confianza por parte de los ciudadanos. El paciente es cada vez más activo, no sólo accede a la información sino que la demanda, comparte, socializa e incluso la crea. Como profesionales, nos vemos limitados por la presión asistencial, la preocupación por la privacidad, la inercia de la presencialidad y la falta de formación. Ubicando al paciente en el centro del sistema, se abren nuevos retos en atención sanitaria. En este contexto, el blog destinado a enfermos renales, trata de responder a las inquietudes y demandas del momento, dando un valor añadido a la consulta de Nefrología. Son los primeros pasos de un largo camino en el que pacientes y profesionales hemos de ir generando, sumando y compartiendo conocimiento que contribuya a mejorar la calidad de vida de las personas con insuficiencia renal crónica. 


\section{Referencias Bibliográficas}

1. El ePaciente y las redes sociales - Salud 2.0. [Internet]. Fundacion Vodafone: Traver Salcedo, V. y Fernández Luque, L. Edita: Publidisa Julio 2011.ISBN: 978-84-694-4110-7. Disponible en: http://www.vodafone.es/static/fichero/pro_ucm _ mgmt_017600.pdf Visitado: septiembre 2012.

2. CDC's Guide to writing for Social Media. [Internet]. US Department of Health and Human Services. Centers for Disease Control and Prevention. Abril 2012. Disponible en: http://www.cdc.gov/socialmedia/Tools/guidelines/pdf/GuidetoWritingforSocial Media.pdf Visitado: septiembre 2012.

3. Plataforma de profesionales de Osakidetza. Profesionales en la red. [Internet]. Martín Rodriguez, M.A. Mayo 2012. Disponible en: http:// osakidetza.blog.euskadi.net/ezagutza/wpcontent/ themes/ficheros/Presentación http://www.prosakidetza.net/groups/Visitado: octubre 2012.

4. Programa interactivo para pacientes. Hospital Clinic y Fundación BBVA. [Internet]. Disponible en: http://www.forumclinic.org/ Visitado: septiembre 2012.

5. Blog: "Vivir con insuficiencia renal". [Internet]. Disponible en: http://ana-vivirconinsuficienciarenal.blogspot.com.es/Visitado: noviembre2012.

6. Blog. El paciente híbrido. Disponible en: http:// hibridosanitario.wordpress.com/Visitado: septiembre 2012.

7. Social Media Guidelines and Best Practices. [Internet]. Department of Health and Human Services. Centers for Disease Control and Prevention. Division of news and electronic media. Agosto 2011. Disponible en: http://www.cdc.gov/socialmedia/tools/guidelines/pdf/widgetguidelines.pdf. Visitado: octubre 2012.

8. Olalde, R. "Autogestión de la salud a lo largo de la vida". Interacción virtual pacientes y profesionales de la salud". Presentación I Jornada 2012 de la Academia de Ciencias de Enfermería de Bizkaia 29/11/2012. Bilbao.
9. Benito, R. "Autogestión de la salud a lo largo de la vida". Interacción virtual pacientes y profesionales de la salud". Presentación I Jornada 2012 de la Academia de Ciencias de Enfermería de Bizkaia 29/11/2012. Bilbao. Disponible en: http:// es.slideshare.net/rakeltxu78/interaccin-virtualentre-pacientes-y-profesionales.

10. Los ciudadanos ante la e-Sanidad. Julio 2012. [Internet]. Hernando Martín,I, Polo Sanz,P, Vázquez Martínez,R, García Otero,A. Observatorio Nacional de las Telecomunicaciones y de la Sociedad de la Información (ONTSI) y de la Dirección de Programas de la Entidad Pública Empresarial Red.es, del Ministerio de Industria, Energía y Turismo. [Internet]. Disponible en: http://www.ontsi.red.es/ontsi/sites/ default/files/pr_ciudadanos_esanidad.pdf http://www. ontsi.red.es/ontsi/sites/default/files/informe ciudadanos_esanidad.pdf Visitado: noviembre 2012.

11. Programa de acreditación de páginas web sanitarias. Agencia de calidad sanitaria de Andalucía. [Internet]. Junio 2011. [Internet]. Disponible en: http://www.calidadwebsalud.com/ Visitado: noviembre 2012.

12. Alejandro Jadad R, Carlos Rizo A, Murray W Enkin. I am a good patient, believe it or not. BMJ 2003;326:1293 (Published 12 June 2003). [Internet]. Disponible en: http://www.webcitation.org/5tIgwWaVP http://www.bmj.com/content/326/7402/1293.full Visitado: Enero 2013.

13. 12 indicadores efectivos para medir el éxito de tu blog. Puro Marketing. Diario Digital Líder de Marketing, Publicidad y Social Media en Español. [Internet]. 23-11-201l. Disponible en: http://www. puromarketing.com/10/11422/indicadores-efectivos-para-medir-exito-blog.html Visitado: marzo 2013.

14. La Red Vasca de pacientes. Kronikoen Sarea. 2012. Departamento de Sanidad y Consumo del Gobierno Vasco. [Internet]. Disponible en: https://www.kronikoensarea.net/Visitado: Septiembre 2012.

15. Información y recursos para pacientes. 09/2012. [Internet]. http://www.cuidando.org/ Visitado: febrero 2013. 